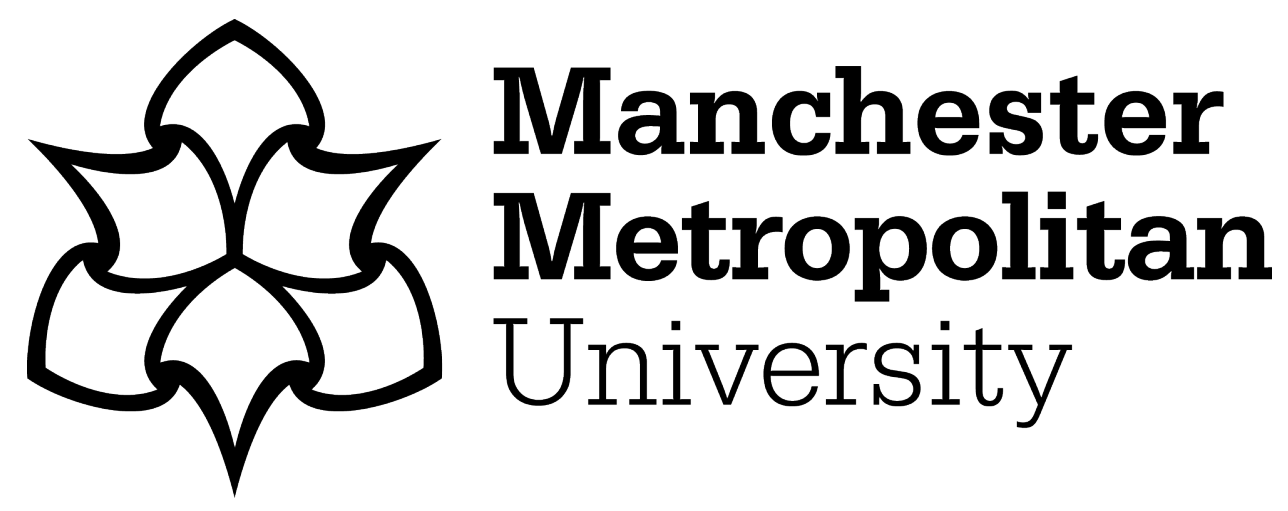

Rushton, Alison, Evans, David, Middlebrook, Nicola ORCID logoORCID: https://orcid.org/0000-0003-2154-5723, Heneghan, Nicola and Falla, Deborah (2017) An innovative approach to understanding mechanisms in the transition from acute to chronic pain in a musculoskeletal trauma population. In: Physiotherapy UK Conference 2017, 10 November 2017 - 11 November 2017, Birmingham, UK.

Downloaded from: https://e-space.mmu.ac.uk/627790/

Version: Accepted Version

DOI: https://doi.org/10.1016/j.physio.2017.11.106

Please cite the published version 


\section{An innovative approach to understanding mechanisms in the transition from acute to chronic pain in a musculoskeletal trauma population}

Purpose: Pain is a common and expected sequela of musculoskeletal trauma, yet there is poor understanding as to why some patients develop chronic and disabling post-traumatic pain. Rehabilitation is widely regarded as an important component of post-trauma healthcare however, the current position of equipoise means that precision rehabilitation has not yet been achieved. Understanding mechanisms that underlie the transition from acute to chronic pain is essential to moving beyond this position, yet no research to date has comprehensively measured the activity of pertinent mechanisms in a prognostic study in this population. With evidence that precision rehabilitation can improve effectiveness of pain management, identifying prognostic factors related to long-term pain and disability outcome may facilitate targeting of effective interventions. The aim of this presentation is to present a unique and comprehensive combination of patient reported outcome measures, psychophysical testing and biomarkers which will be applied to help elucidate the transition from acute to chronic pain following musculoskeletal trauma.

Methods: To understand transition to chronicity, outcome measures relating to the four primary mechanisms of pain are required, specifically: nociceptive (injury location, severity and characteristics), neuropathic (painDETECT tool and pain extent), inflammatory (biomarkers), and central hypersensitivity (quantitative sensory testing, painDETECT and pain extent). Concurrently, patient-reported outcome measures are required to assess general health and psychosocial factors (e.g. EQ-5D-5L, Hospital Anxiety and Depression Scale), which are likely to influence pain and disability. Choosing an outcome to measure long-term pain recovery is a key but difficult decision for a post-trauma population, owing to likely clinical heterogeneity. The Chronic Pain Grade Scale, with its established measurement properties, is the best measure to capture both pain and disability. Results: A prognostic study to gain a comprehensive baseline profile of acute post-trauma pain mechanisms using a variety of measures (patient reported outcome measures, psychophysical testing and biomarkers), and evaluate their 
relationships to long-term pain and disability is required. This will allow us, for the first time, to (1) develop and validate a prognostic tool to predict development of chronic and disabling pain (2) begin the process of targeting precision rehabilitation interventions.

Conclusion(s): There is a need for a prognostic study of this kind to comprehensively evaluate the primary mechanisms of pain to predict transition from acute to chronic pain in a musculoskeletal trauma population. This understanding is crucial to optimising physiotherapy practice through precision rehabilitation.

Implications: Understanding predictors of long term pain and disability will support the development of a prediction tool. This will inform rehabilitation decision making, and facilitate improvements in clinical and cost effectiveness. 